\title{
Design of nanoporous materials with optimal sorption capacity
}

\author{
Xuan Zhang, ${ }^{1}$ Koki Urita, ${ }^{2}$ Isamu Moriguchi, ${ }^{2}$ and Daniel M. Tartakovsky ${ }^{1, a)}$ \\ ${ }^{1}$ Department of Mechanical and Aerospace Engineering, University of California, San Diego, \\ 9500 Gilman Drive, La Jolla, California 92093, USA \\ ${ }^{2}$ Department of Applied Chemistry, Graduate School of Engineering, Nagasaki University, \\ Nagasaki 852-8521, Japan
}

(Received 11 May 2015; accepted 16 June 2015; published online 25 June 2015)

\begin{abstract}
Modern technological advances have enabled one to manufacture nanoporous materials with a prescribed pore structure. This raises a possibility of using controllable pore-scale parameters (e.g., pore size and connectivity) to design materials with desired macroscopic properties (e.g., diffusion coefficient and adsorption capacity). By relating these two scales, the homogenization theory (or other upscaling techniques) provides a means of guiding the experimental design. To demonstrate this approach, we consider a class of nanoporous materials whose pore space consists of nanotunnels interconnected by nanotube bridges. Such hierarchical nanoporous carbons with mesopores and micropores have shown high specific electric double layer capacitances and high rate capability in an organic electrolyte. We express the anisotropic diffusion coefficient and adsorption coefficient of such materials in terms of the tunnels' properties (pore radius and inter-pore throat width) and their connectivity (spacing between the adjacent tunnels and nanotube-bridge density). Our analysis is applicable for solutes that undergo a nonequilibrium Langmuir adsorption reaction on the surfaces of fluid-filled pores, but other homogeneous and heterogeneous reactions can be handled in a similar fashion. The presented results can be used to guide the design of nanoporous materials with optimal permeability and sorption capacity. (C) 2015 AIP Publishing LLC. [http://dx.doi.org/10.1063/1.4923057]
\end{abstract}

\section{INTRODUCTION}

Small pore sizes and large surface porosity are some of the unique properties of nanoporous materials, which can be used in a wide range of applications involving ion exchange, adsorption, sensing, and catalysis. ${ }^{1-3}$ Nanoporous materials play central role in biomedical diagnostics, such as combinatorial biochemistry on-a-chip, chromatography, biosensors, cell manipulation, and DNA analysis. ${ }^{4-6}$ Because of their high electrical conductivity and wettability towards electrolytes, nanoporous materials might significantly boost the performance of energy storage devices. ${ }^{7-9}$

Desirable properties of manufactured (nano)porous materials can be enhanced by controlling their pore structure and physicochemical properties of emerging pore networks. ${ }^{10}$ For instance, introduction of active sites onto pore surfaces of mesoporous metallosilicate materials is a promising venue for designing novel adsorbents and catalysis. ${ }^{11}$ Embedment of microporous tubes ${ }^{12}$ between layers of macro- and nanopores results in new materials with superior mechanical and thermal properties. ${ }^{13}$ For example, hierarchical nanoporous carbons with mesopores and micropores have shown high specific electric double layer capacitances and high rate capability in an organic electrolyte.

Quantitative predictions of macroscopic properties of such new materials (e.g., their sorption capacity or thermal conductivity) require the ability to relate these properties to their pore-scale counterparts, such as a pore-size distribution and pore-network connectivity. ${ }^{14}$ This can be accomplished

${ }^{a)}$ Electronic mail: dmt@ucsd.edu with a variety of upscaling techniques, including volume averaging $^{15}$ and homogenization via multiple-scale expansions. ${ }^{16-18}$ We use the latter approach to predict anisotropic diffusion coefficients and effective adsorption rates for a class of nanoporous materials, ${ }^{19}$ whose synthesis allows one to control their pore structure. It is worthwhile emphasizing that the use of various homogenization techniques to derive effective (continuum- or Darcy-scale) models of reactive transport in porous media (primarily subsurface environments) is not new. ${ }^{20-23}$ The novelty of our study comes from using such techniques to optimize macroscopic properties of a material by using its microscopic properties as decision variables.

The effective reaction rate in a given solid-solution system is an essential factor for sorption-system design. Our analysis is applicable for solutes that undergo a non-equilibrium Langmuir-type adsorption reaction on the surfaces of fluidfilled pores, but other homogeneous and heterogeneous reactions can be handled in a similar fashion. The presented results can be used to guide the design of nanoporous materials with optimal permeability and sorption capacity.

In Sec. II, we formulate a non-equilibrium pore-scale model, which is based on the first-order theory of sorption kinetics. $^{24-26}$ The corresponding continuum-scale model is derived in Sec. III. This model relates the macroscopic properties of a nanoporous material (i.e., its diffusion coefficient and adsorption capacity) to its pore-scale counterparts (e.g., the radii of micro- and macropores, and the size of nanobridges). In Sec. IV, we present simulations results and discuss their implications for material design. Our findings are summarized in Sec. V. 


\section{PROBLEM FORMULATION}

Consider a nanoporous material $\hat{\Omega}$ with a characteristic length $L$. Let $\hat{\mathcal{P}}$ denote the part of this material occupied by nanopores whose characteristic length scale, e.g., a typical pore diameter, is $l$ such that $\epsilon \equiv l / L \ll 1$. The impermeable solid skeleton $\hat{\mathcal{S}}$ occupies the rest of the nanoporous material, i.e., $\hat{\Omega}=\hat{\mathcal{P}} \cup \hat{\mathcal{S}}$. The (multi-connected, smooth) boundary between the pore space $\hat{\mathcal{P}}$ and the solid skeleton $\hat{\mathcal{S}}$ is denoted by $\hat{\Gamma}$.

The pore space $\hat{\mathcal{P}}$ is occupied by a fluid, which contains a solute with concentration $\hat{c}(\hat{\mathbf{x}}, \hat{t})\left[\mathrm{mol} / \mathrm{L}^{3}\right]$, where $\hat{\mathbf{x}}$ denotes a point in $\hat{\mathcal{P}}$ and $\hat{t}$ is time. The solute diffuses throughout the pore space $\hat{\mathcal{P}}$ and undergoes a heterogeneous reaction at the solid-fluid interface $\hat{\Gamma}$, both taking place in isothermal conditions. The former process is described by a diffusion equation

$$
\frac{\partial \hat{c}}{\partial \hat{t}}=D \hat{\nabla}^{2} \hat{c}, \quad \hat{\mathbf{x}} \in \hat{\mathcal{P}}, \quad \hat{t}>0
$$

where $D\left[\mathrm{~L}^{2} / \mathrm{T}\right]$ is the diffusion coefficient. The pore spaces considered in our analysis consist of both interconnected nanopores and nanotubes. The diffusive transport in the former is due to molecular diffusion (with molecular diffusion coefficient $D_{m}$ ), while Knudsen diffusion (with diffusion coefficient $D_{k}$ ) might take place in the latter. While the analysis presented below is capable of handling spatially variable and anisotropic diffusion coefficients, we use the constant diffusion coefficient $D$ to simplify the presentation.

Mass conservation along the impermeable solid-fluid interface $\hat{\Gamma}$ requires the normal component of the solute mass flux $-D \hat{\nabla} \hat{c}$ to be balanced by the rate of change in the adsorbed solute $\hat{q}$, i.e.,

$$
-D \mathbf{n} \cdot \hat{\nabla} \hat{c}=\frac{\partial \hat{q}}{\partial \hat{t}}=\hat{q}_{\mathrm{m}} \frac{\partial \hat{s}}{\partial \hat{t}}, \quad \hat{\mathbf{x}} \in \hat{\Gamma}, \quad \hat{t}>0,
$$

where $\hat{q}\left[\mathrm{~mol} / \mathrm{L}^{2}\right]$ is the adsorption amount per unit area of the solid-fluid surface $\hat{\Gamma}, \hat{q}_{\mathrm{m}}\left[\mathrm{mol} / \mathrm{L}^{2}\right]$ is the maximal adsorption amount, $\hat{s}(\hat{\mathbf{x}}, \hat{t})[-]$ is the fractional coverage of $\hat{\Gamma}$, and $\mathbf{n}(\mathbf{x})$ is the unit normal vector of $\hat{\Gamma}$. Equation (1) is also subject to an initial condition $\hat{c}(\mathbf{x}, 0)=c_{\text {in }}$, in which the initial concentration $c_{\text {in }}$ assumed to be spatially uniform. Finally, appropriate boundary conditions are imposed on the bounding surface of the nanoporous material $\hat{\Omega}$.

Previous efforts to derive effective (continuum-scale) descriptions of processes, which admit the pore-scale representations (1) and (2), dealt with either equilibrium adsorption $^{27,28}$ or general non-equilibrium adsorption described by a coupled system of reaction-diffusion equations for $\hat{c}$ and $\hat{s} .^{20-23,29}$ While the former are not applicable for many nanoporous phenomena, the latter are too complex to be used in materials design. Instead, we derive a reduced-complexity model based on the Lagergren (pseudo-first-order) rate equation ${ }^{30-32}$

$$
\frac{\mathrm{d} \hat{s}}{\mathrm{~d} \hat{t}}=\gamma\left(\hat{s}_{\mathrm{eq}}-\hat{s}\right),
$$

where $\gamma\left(\mathrm{T}^{-1}\right)$ is the first-order adsorption rate constant and $\hat{s}_{\text {eq }}$ is the adsorption coverage fraction at equilibrium. The latter is assumed to follow the Langmuir adsorption isotherm

$$
\hat{s}_{\mathrm{eq}}=\frac{\hat{K} \hat{c}}{1+\hat{K} \hat{c}},
$$

where $\hat{K}\left(\mathrm{~L}^{3} / \mathrm{mol}\right)$ is the adsorption equilibrium constant. The derivation of the reduced-complexity model is facilitated by rewriting this pore-scale boundary-value problem (BVP) in the dimensionless form. Let us introduce dimensionless variables and parameters

$$
\begin{aligned}
& \mathbf{x}=\frac{\hat{\mathbf{x}}}{L}, \quad t=\frac{D \hat{t}}{L^{2}}, \quad c=\frac{\hat{c}}{c_{\mathrm{in}}}, \quad q_{\mathrm{m}}=\frac{\hat{q}_{\mathrm{m}}}{L c_{\mathrm{in}}}, \\
& K=\hat{c}_{\mathrm{in}} \hat{K}, \quad \mathrm{Da}=\frac{L^{2} \gamma}{D},
\end{aligned}
$$

where the Damköhler number Da represents the ratio of reaction and diffusion time-scales. We show in Appendix A that, for nanoporous materials characterized by $\mathrm{Da} \ll 1$, Eqs. (2)-(4) give rise to a nonlinear (but decoupled) boundary condition at the solid-fluid interface $\Gamma$

$$
-\mathbf{n} \cdot \nabla c=\operatorname{Da} q_{\mathrm{m}} \frac{K c}{1+K c}, \quad \mathbf{x} \in \Gamma, \quad t>0
$$

The remainder of the pore-scale BVP takes the form of a diffusion equation

$$
\frac{\partial c}{\partial t}=\nabla^{2} c, \quad \mathbf{x} \in \mathcal{P}, \quad t>0
$$

subject to the initial condition $c(\mathbf{x}, 0)=1$ for $\mathbf{x} \in \mathcal{P}$ and the corresponding boundary conditions on the bounding surface of the nanoporous material $\Omega$.

Solving this BVP in a large interconnected network of nanopores, $\mathcal{P}$, is neither feasible nor necessary. Instead, one is often interested in the emerging (macroscopic) properties of the nanoporous material $\Omega$ as a whole. While its pore structure is described by diameters and a spatial arrangement of individual nanopores and nanotubes bridges, its macroscopic properties are characterized by bulk (average) quantities such as porosity $\phi$, effective diffusion coefficient $\mathbf{D}_{\text {eff }}$, and effective adsorption rate constant $\gamma_{\text {eff }}$. Connecting these two levels of description allows us to relate such macroscopic properties to the underlying microscopic (pore-scale) characteristics. This, in turn, enables one to manufacture nanoporous materials with desired macroscopic attributes.

By way of example, we consider nanoporous materials in which rows of interconnected uniform nanopores of radius $R$ are bridged by regularly placed microporous tubes. The spacing between two neighboring pores in a single row is expressed in terms of the angle $\theta$ that we refer to as an intersection factor. A number $n$ of microporous tubes (diameter $d=0.7 \mathrm{~nm}$ ) per unit cell is referred to as a microporous tube density. In the first class of materials, the rows of interconnected nanopores are perfectly aligned (Fig. 1). In the second, the rows are shifted relative to each other by the distance $R / 2$ (Fig. 6). These two settings provide an adequate representation of the nanoporous materials. ${ }^{19}$ 

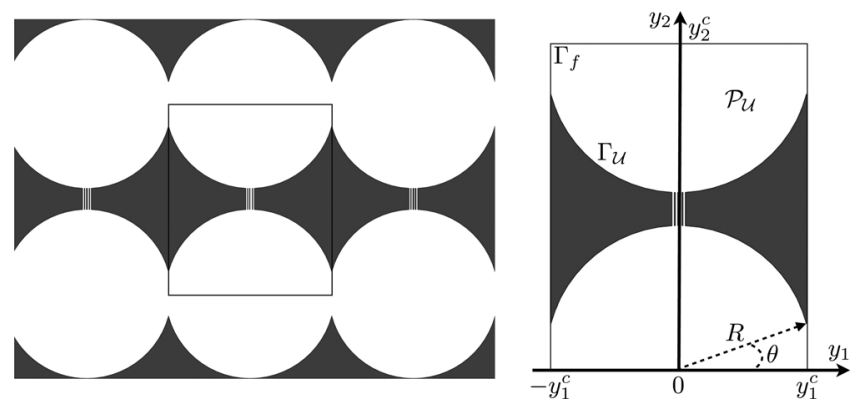

FIG. 1. A schematic representation of Material 1 (left) and the corresponding unit cell $\mathcal{U}$ (right). The unit cell is a $2 y_{1}^{c} \times y_{2}^{c}$ rectangle, with $y_{1}^{c}=$ $R \cos \theta$ and $y_{2}^{c}=2 R+l$.

\section{MACROSCOPIC PROPERTIES OF NANOPOROUS MATERIALS}

Macroscopic representations of a nanoporous material $\Omega$ treat it as a continuum, without separating it into the pore space $\mathcal{P}$ and the solid skeleton $\mathcal{S}$. Macroscopic solute concentration $C(\mathbf{x}, t)$ - the microscopic concentration $c(\mathbf{y}, t)$ averaged over a (representative elementary) volume $V$ centered at point $\mathbf{x} \in \Omega$-is defined as

$$
C(\mathbf{x}, t) \equiv \frac{1}{\left\|\mathcal{P}_{V}\right\|} \int_{\mathcal{P}_{V}(\mathbf{x})} c(\mathbf{y}, t) \mathrm{d} \mathbf{y},
$$

where $\left\|\mathcal{P}_{V}\right\|$ is the total volume of pores contained in $V$.

We show in Appendix B that, for nanoporous materials composed of periodic arrangements of unit cells $\mathcal{U}$, the macroscopic concentration $C(\mathbf{x}, t)$ satisfies a reaction-diffusion equation

$$
\frac{\partial C}{\partial t}=\phi^{-1} \nabla \cdot\left(\mathbf{D}_{\mathrm{eff}} \nabla C\right)-\gamma_{\mathrm{eff}} q_{\mathrm{m}} \frac{K C}{1+K C},
$$

where $\phi \equiv\left\|\mathcal{P}_{V}\right\| /\|V\|=\left\|\mathcal{P}_{\mathcal{U}}\right\| /\|\mathcal{U}\|$ is the porosity (with $\mathcal{P}_{\mathcal{U}}$ denoting the pore space of the unit cell $\mathcal{U}$ ); the effective reaction constant $\gamma_{\text {eff }}$ is related to $\left\|\Gamma_{V}\right\|$, the total surface area of the pores contained in $V$ (or to $\left\|\Gamma_{\mathcal{U}}\right\|$, the total surface area of the pores contained in $\mathcal{U}$ ) by

$$
\gamma_{\mathrm{eff}}=\frac{\left\|\Gamma_{V}\right\|}{\left\|\mathcal{P}_{V}\right\|}=\frac{\left\|\Gamma_{\mathcal{U}}\right\|}{\left\|\mathcal{P}_{\mathcal{U}}\right\|}
$$

and the effective diffusion tensor $\mathbf{D}_{\text {eff }}$ defined as

$$
\mathbf{D}_{\text {eff }}=\frac{D}{\|\mathcal{U}\|} \int_{\mathcal{P}_{\mathcal{U}}}\left(\mathbf{I}+\nabla_{\mathbf{y}} \chi\right) \mathrm{d} \mathbf{y} .
$$

Here, $\mathbf{I}$ is the identity matrix, and the "closure variable" $\chi(\mathbf{y})$ is a $\mathcal{U}$-periodic vector defined on $\mathcal{P}_{\mathcal{U}}$. It satisfies

$$
\nabla_{\mathbf{y}}^{2} \chi=0, \quad \mathbf{y} \in \mathcal{P}_{\mathcal{U}} ; \quad\langle\chi(\mathbf{y})\rangle \equiv \frac{1}{\|\mathcal{U}\|} \int_{\mathcal{P}_{\mathcal{U}}} \chi(\mathbf{x}) \mathrm{d} \mathbf{y}=0
$$

subject to the boundary condition along the fluid-solid segments $\Gamma_{\mathcal{U}}$ of the boundary of $\mathcal{P}_{\mathcal{U}}$

$$
\mathbf{n} \cdot \nabla_{\mathbf{y}} \chi=-\mathbf{n} \cdot \mathbf{I}, \quad \mathbf{y} \in \Gamma_{\mathcal{U}} .
$$

Along the remaining ("fluid") segments $\Gamma_{f}$ of the boundary of $\mathcal{P}_{\mathcal{U}}$ (see Fig. 1), the $\mathcal{U}$-periodicity of $\chi(\mathbf{x})$ is enforced. Accounting for the normalization condition $\langle\chi(\mathbf{y})\rangle=0$, in the case of the rectangular unit cell $\mathcal{U}$ (Fig. 1), this yields

$$
\begin{aligned}
\chi_{1}\left(-y_{1}^{c}, y_{2}\right) & =\chi_{1}\left(y_{1}^{c}, y_{2}\right)=0, \\
\frac{\partial \chi_{1}}{\partial y_{2}}\left(y_{1}, 0\right) & =\frac{\partial \chi_{1}}{\partial y_{2}}\left(y_{1}, y_{2}^{c}\right)=0
\end{aligned}
$$

and

$$
\begin{gathered}
\chi_{2}\left(y_{1}, 0\right)=\chi_{2}\left(y_{1}, y_{2}^{c}\right)=0, \\
\frac{\partial \chi_{2}}{\partial y_{1}}\left(-y_{1}^{c}, y_{2}\right)=\frac{\partial \chi_{2}}{\partial y_{1}}\left(y_{1}^{\mathrm{c}}, y_{2}\right)=0 .
\end{gathered}
$$

Here, $y_{1}^{c}=R \cos \theta$ and $y_{2}^{c}=2 R+l$. Figure 2 exhibits a numerical solution $\chi(\mathbf{y})=\left(\chi_{1}, \chi_{2}\right)^{\top}$ of the BVP (11)-(13) defined on $\mathcal{P}_{\mathcal{U}}$ in Fig. 1. This solution and all the numerical results reported below are obtained with COMSOL.

\section{SIMULATION RESULTS}

The upscaled relations derived above allow one to infer macroscopic properties of a nanomaterial, e.g., the (effective) anisotropic diffusion coefficient $\mathbf{D}_{\text {eff }}$ and the effective reaction constant $\gamma_{\text {eff }}$, from its microscopic characteristics. Unless specified otherwise, the subsequent simulations deal with the effects of these microscopic parameters on the macroscopic properties of Material 1 (Fig. 1). The external concentration gradient is applied in the $x_{1}$ direction.
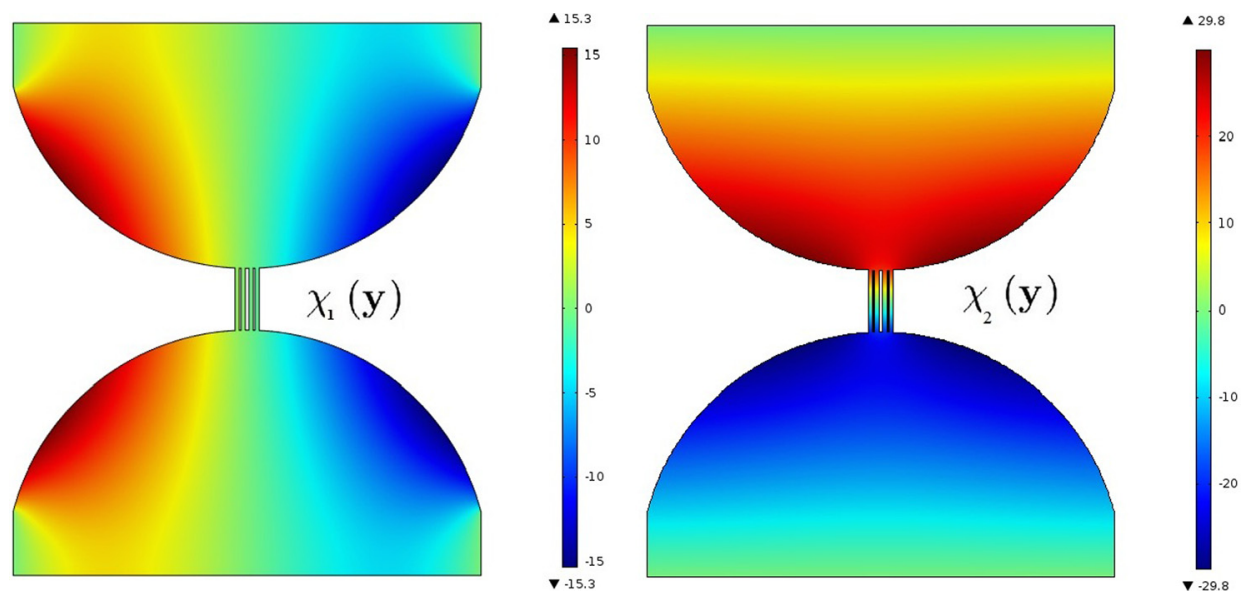

FIG. 2. The horizontal $\chi_{1}$ (left) and vertical $\chi_{2}$ (right) components of the closure variable $\chi(\mathbf{y})=\left(\chi_{1}, \chi_{2}\right)^{\top}$ defined on the fluid-domain $\mathcal{P}_{\mathcal{U}}$ of the unit cell $\mathcal{U}$ in Fig. 1 . In this example, the four vertical nanobridges are spaced $\Delta=0.3 \mathrm{~nm}$ apart. 

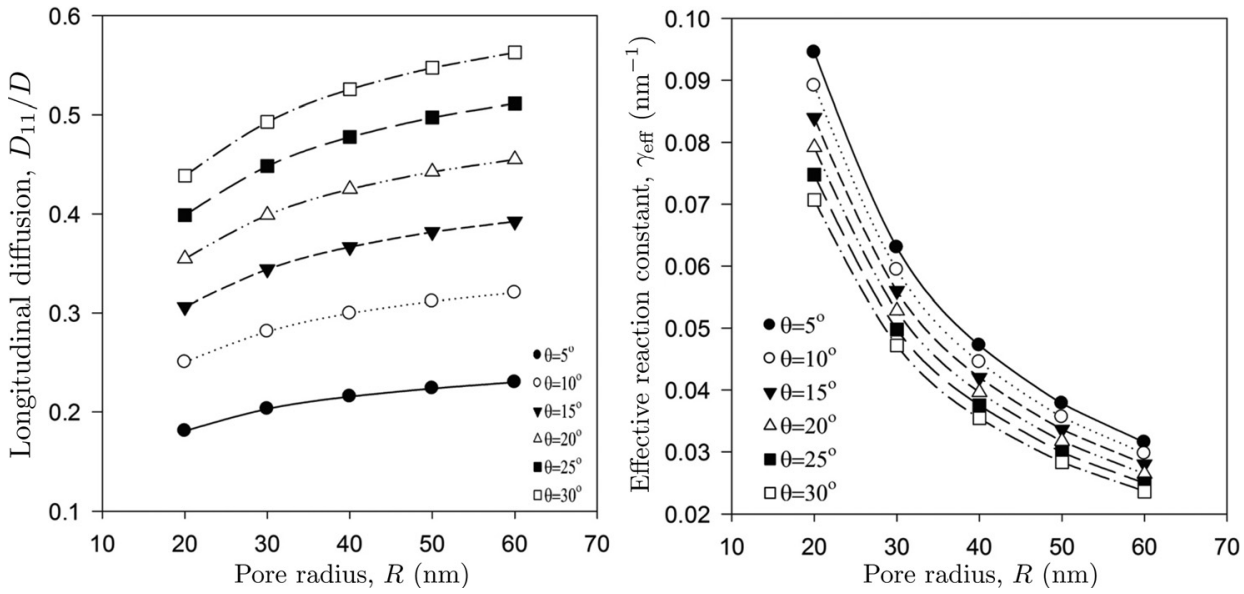

FIG. 3. Dependence of effective longitudinal diffusion coefficient $D_{11}$ (left) and effective reaction constant $\gamma_{\text {eff }}$ (right) on pore radius $R$ and intersection angle $\theta$.

\section{A. Nanopore radius in isolated nanotunnels}

In the absence of nanobridges $(n=0)$, diffusion through the nanoporous Material 1 (Fig. 1) occurs only in the $x_{1}$ direction along the disconnected nanotunnels. The latter consist of stacked nanopores of uniform radius $R$, which varies from $R=20 \mathrm{~nm}$ to $60 \mathrm{~nm}$. The effective (macroscopic) longitudinal diffusion coefficient $D_{11}$, normalized by molecular diffusion coefficient $D$, and the effective reaction constant $\gamma_{\text {eff }}$ are shown in Fig. 3; the transverse diffusion coefficient is $D_{22}=0$. The effective diffusion coefficient $D_{11}$ increases with pore radius $R$ and intersection angle $\theta$. The reaction constant $\gamma_{\text {eff }}$ decreases with both.

\section{B. Intersection angle $\theta$}

Intersection angle $\theta$ quantifies the distance between the centers of any two adjacent spherical pores in a given nanotunnel (Fig. 1), such that $\theta$ decreases as this distance increases. Figure 4 exhibits the impact of $\theta$ on the effective properties of Material 1 (Fig. 1), in which nanotunnels composed of pores of radius $R=40 \mathrm{~nm}$ are connected by microporous tubes of length $l=10 \mathrm{~nm}$. As $\theta$ increases, the throats between the adjacent pores become large, giving rise to a significant increase in the longitudinal component of the effective diffusion tensor $\left(D_{11}\right)$. The impact of $\theta$ on both the transverse diffusion $\left(D_{22}\right)$ and the effective reaction constant $\left(\gamma_{\text {eff }}\right)$ is less pronounced. A slight $(\approx 3 \%)$ increase of the longitudinal diffusion coefficient $D_{11}$ caused by the addition of $n=10$ nanotubes is due to the rise in the material's connectivity and porosity. Despite their negligible contribution to the porosity of this nanoporous material, the microporous tube bridges significantly alter its diffusive properties, giving rise to the non-zero transverse diffusion coefficient $D_{22}$. They also nearly double the effective reaction constant $\gamma_{\text {eff }}$.

\section{Nanobridge density}

This numerical experiment deals with nanotunnels formed by pores of radius $R=40 \mathrm{~nm}$, which are intersected at angle $\theta=20^{\circ}$; the neighboring nanotunnels are connected by microporous tubes of length $l=10 \mathrm{~nm}$. In the simulations reported below, we set the distance between individual microporous tubes to either $\Delta=1.4 \mathrm{~nm}$ or $\Delta=0.3 \mathrm{~nm}$. For the given microporous tube length of $l=10 \sim 11 \mathrm{~nm}$ and $\Delta=$ $0.3 \mathrm{~nm}$, the maximum possible number of microporous tubes in the unit cell is $n_{\max }=10$. If no limits are placed on the microporous tube length (i.e., if it is allowed to vary between $l=10 \sim 50 \mathrm{~nm}$ ), then placing microporous tubes $\Delta=1.4$ $\mathrm{nm}$ apart would allow for the maximum number of microporous tubes in the unit cell is $n_{\max }=34$. The behavior of the effective transport properties of these nanoporous materials as a function of nanobridge density is shown in Fig. 5. While the number of microporous tubes in a bridge, $n$, does not appreciably affect the material's diffusive properties $\left(D_{11}\right.$ and $\left.D_{22}\right)$, it significantly influences its adsorbing capacity $\left(\gamma_{\text {eff }}\right)$.

\section{Alternative material topology}

To investigate the effects of spatial arrangement of nanotunnels and nanobridges comprising a nanoporous material, we consider the second class of materials (Material 2
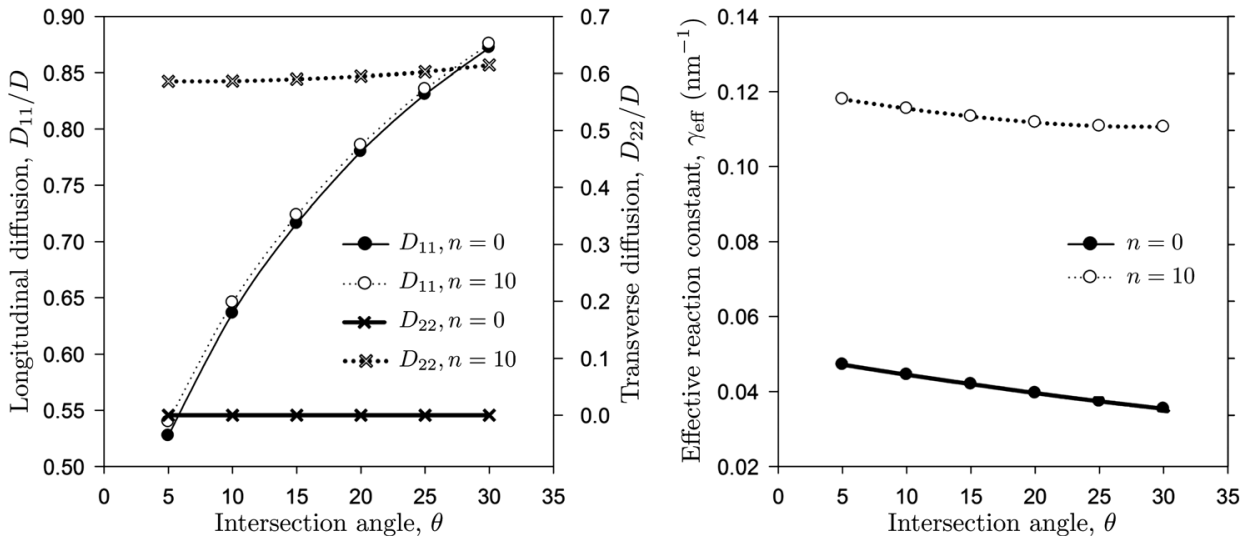

FIG. 4. Dependence of the longitudinal $\left(D_{11}\right)$ and transverse $\left(D_{22}\right)$ components of the effective diffusion tensor (left) and effective reaction constant $\gamma_{\text {eff }}$ (right) on intersection angle $\theta$, for materials with disconnected $(n=0)$ and interconnected (by $n=10$ microporous tubes) nanotunnels. The latter are composed of pores with uniform radius $R=40 \mathrm{~nm}$. 

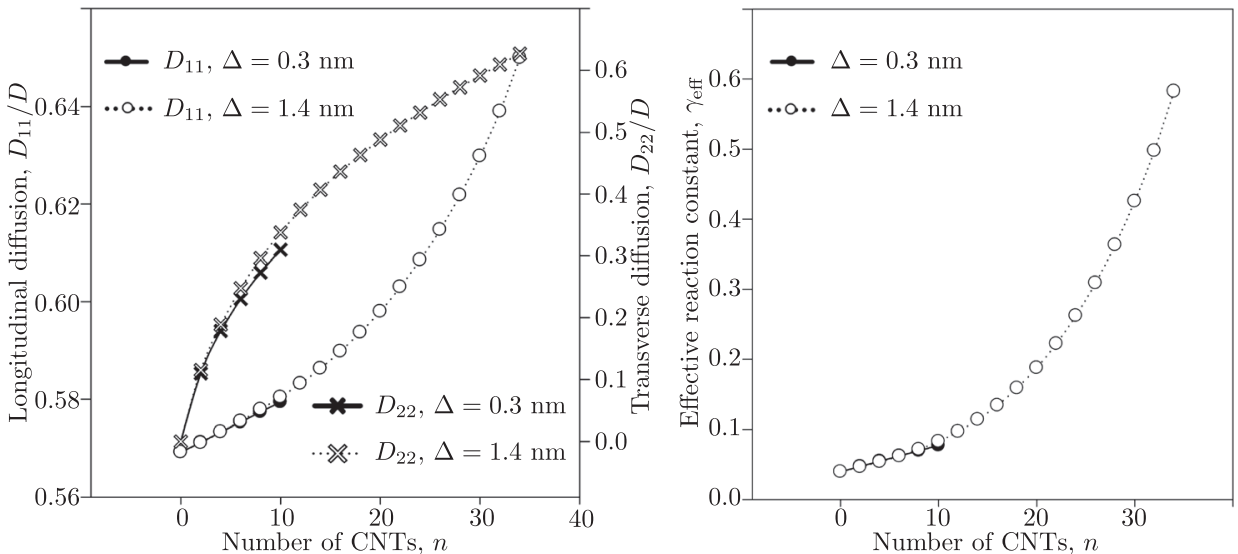

FIG. 5. Dependence of the longitudinal $\left(D_{11}\right)$ and transverse $\left(D_{22}\right)$ components of the effective diffusion tensor (left) and effective reaction constant $\gamma_{\text {eff }}$ (right) on the number of microporous tubes $(n)$ comprising the nanobridges between the neighboring nanotunnels. The latter are composed of pores with uniform radius $R=40 \mathrm{~nm}$, which are intersected at angle $\theta=20^{\circ}$.

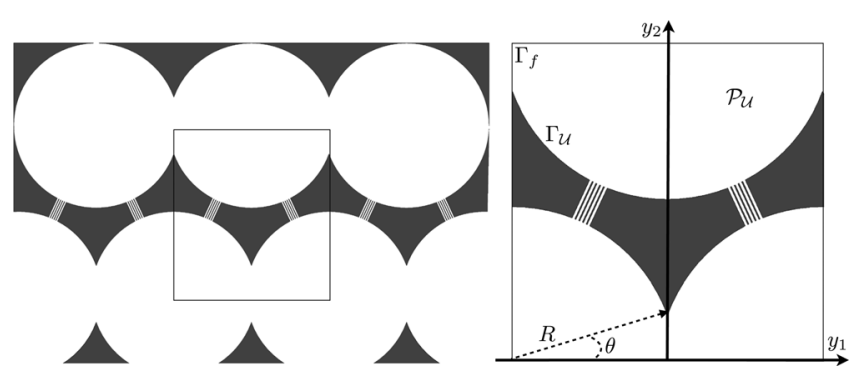

FIG. 6. A schematic representation of Material 2 and the corresponding unit cell $\mathcal{U}$ (right).

in Fig. 6). The neighboring nanotunnels in these materials are shifted by $R / 2$, while in Material 1 (Fig. 1) they are aligned.

For a given structure of the nanotunnels (e.g., the pore radius $R=40 \mathrm{~nm}$ and the intersection angle $\theta=20^{\circ}$ ) and nanobridges (e.g., the microporous tube spacing $\Delta=0.3 \mathrm{~nm}$ and the number of microporous tubes $n$ ), Eq. (9) suggests that Materials 1 and 2 have the same adsorbing capacity (the effective reaction constant $\gamma_{\text {eff }}$ ). The topologic differences between the two materials manifest themselves in the macroscopic diffusive properties (Fig. 7). Material 2 possesses the higher longitudinal diffusion coefficient $\left(D_{11}\right)$ that is

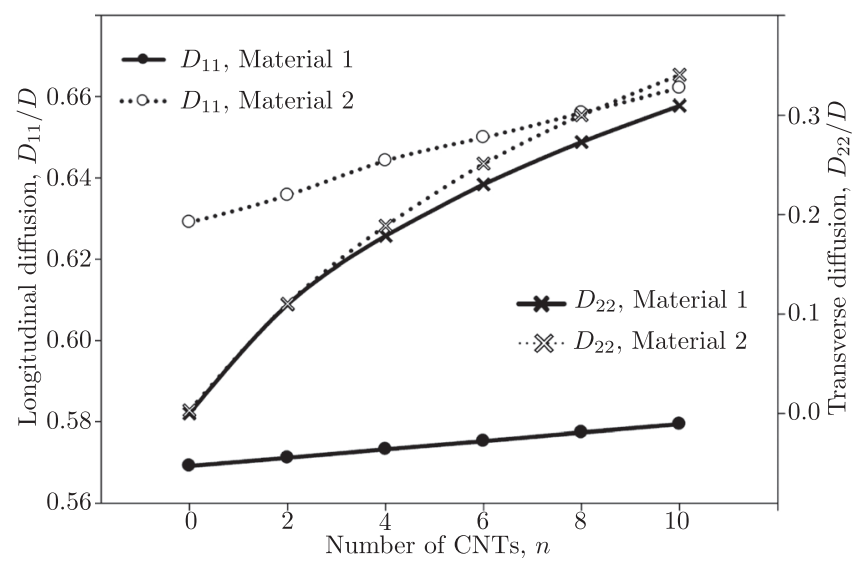

FIG. 7. Dependence of the longitudinal $\left(D_{11}\right)$ and transverse $\left(D_{22}\right)$ components of the effective diffusion tensor on the number of microporous tubes (n) for Materials 1 and 2. In both materials, pores of uniform radius $R=40 \mathrm{~nm}$ are intersected at angle $\theta=20^{\circ}$. significantly more sensitive to the number of microporous tubes in the nanobridge; the transverse diffusion coefficients $\left(D_{22}\right)$ of the two materials are practically the same. Although not shown here, a local distribution of microporous tubes within a unit cell also affects a material's diffusive properties. A symmetric arrangement of nanobridges, which consists of an equal number of nanotubes arranged at acute and obtuse angles (as in Fig. 6), yields the higher values of $D_{11}$ and $D_{22}$ than its asymmetric counterpart, in which only the left or the right bridge is present, does.

\section{CONCLUSIONS}

Modern technological advances have enabled one to manufacture nanoporous materials with a prescribed pore structure. This raises a possibility of using controllable porescale parameters (e.g., pore size and connectivity) to design materials with desired macroscopic properties (e.g., diffusion coefficient and adsorption capacity). By relating these two scales, the homogenization theory (or other upscaling techniques) provides a means of guiding the experimental design. To demonstrate this approach, we considered a class of nanoporous materials whose pore space consists of nanotunnels interconnected by carbon nanotubes (microporous tubes). We express the anisotropic diffusion coefficient and adsorption coefficient of such materials in terms of the tunnels' properties (pore radius and inter-pore throat width) and their connectivity (spacing between the adjacent tunnels and microporous tube-bridge density). Our analysis leads to the following major conclusions.

(1) Upscaling (e.g., by means of the homogenization theory) provides a general framework for optimal design of nanoporous materials. In the case of adsorption-diffusion systems, this approach provides a venue for manufacturing nanoporous materials with desired anisotropic diffusion coefficient $\mathbf{D}_{\text {eff }}$ and reaction constant $\gamma_{\text {eff }}$, which can be used, for example, in the design of filters.

(2) Intersection angle $\theta$ quantifies the distance between the centers of any two adjacent spherical pores in a given nanotunnel. As $\theta$ increases, the throats between the adjacent pores become larger, giving rise to a significant increase in the longitudinal component of the effective diffusion tensor $\left(D_{11}\right)$. The impact of $\theta$ on both the 
transverse diffusion $\left(D_{22}\right)$ and the effective reaction constant $\left(\gamma_{\text {eff }}\right)$ is less pronounced.

(3) Despite their negligible contribution to the porosity of this nanoporous material, the microporous tube bridges significantly alter its diffusive properties, giving rise to the non-zero transverse diffusion coefficient $D_{22}$. They also nearly double the effective reaction constant $\gamma_{\text {eff }}$.

(4) While the number of microporous tubes in a bridge, $n$, does not appreciably affect the material's diffusive properties $\left(D_{11}\right.$ and $\left.D_{22}\right)$, it significantly influences its adsorbing capacity $\left(\gamma_{\text {eff }}\right)$.

\section{ACKNOWLEDGMENTS}

This research was supported in part by Air Force Office of Scientific Research (DE-FG02-07ER25815) and National Science Foundation (EAR-1246315).

\section{APPENDIX A: DERIVATION OF THE BOUNDARY CONDITION}

Rewriting (2) and (3) in terms of the dimensionless quantities (5) yields

$$
-\mathbf{n} \cdot \nabla c=q_{\mathrm{m}} \frac{\partial s}{\partial t}, \quad \text { and } \quad \frac{\mathrm{d} s}{\mathrm{~d} t}=\mathrm{Da}\left(s_{\mathrm{eq}}-s\right) .
$$

In a typical application of nanoporous materials, e.g., mesoporous catalyst membranes, the characteristic macroscopic length (membrane size) is ${ }^{33} L \sim 100 \mu \mathrm{m}$ and the characteristic pore scale is ${ }^{11} l \sim 20-100 \mathrm{~nm}$. Hence, the length-scale ratio is $\epsilon \equiv l / L \sim 10^{-4}$. Moreover, the characteristic time scale of adsorption processes in mesoporous membranes, e.g., for methylene blue, is ${ }^{11,34} 1 / \gamma \sim 40-80 \mathrm{~min}$, while its diffusion coefficient is ${ }^{35} D \sim 10^{-5} \mathrm{~cm}^{2} \mathrm{~s}^{-1}$. Hence, the Damköhler number is $\mathrm{Da} \sim \mathcal{O}\left(10^{-3}\right)$, which allows its use as a small perturbation parameter.

Consider an asymptotic expansion of $s(\mathbf{x}, t)$ in the powers of $\mathrm{Da}, s=s_{0}+\mathrm{Da} s_{1}+\mathcal{O}\left(\mathrm{Da}^{2}\right)$. Substituting this expansion into the second equation in (A1) and collecting the terms of equal powers of Da yields

$$
\frac{\mathrm{d} s_{0}}{\mathrm{~d} t}=0 \quad \text { and } \quad \frac{\mathrm{d} s_{1}}{\mathrm{~d} t}=s_{\mathrm{eq}}-s_{0} .
$$

For the homogenous initial condition, $s(\mathbf{x}, 0)=0$, this yields $s_{0}=0$ and $\mathrm{d} s_{1} / \mathrm{d} t=K c /(1+K c)$. Hence, the first-order approximation of the sorbed concentration, $s \approx s_{0}+\operatorname{Da} s_{1}$, gives rise to

$$
\frac{\mathrm{d} s}{\mathrm{~d} t} \approx \mathrm{Da} \frac{K c}{1+K c} .
$$

Substituting this approximation into (A1) yields (6a).

\section{APPENDIX B: HOMOGENIZATION OF TRANSPORT EQUATIONS}

Three types of local average of a quantity $A(\mathbf{x}, t)$ are defined

$$
\begin{aligned}
\langle A\rangle & \equiv \frac{1}{\|\mathcal{U}\|} \int_{\mathcal{P}_{\mathcal{U}}(\mathbf{x})} A \mathrm{~d} \mathbf{y}, \quad\langle A\rangle_{\mathcal{P}} \equiv \frac{1}{\left\|\mathcal{P}_{\mathcal{U}}\right\|} \int_{\mathcal{P}_{\mathcal{U}}(\mathbf{x})} A \mathrm{~d} \mathbf{y}, \\
\langle A\rangle_{\Gamma} & \equiv \int_{\Gamma_{\mathcal{U}}(\mathbf{x})} A \mathrm{~d} \mathbf{y},
\end{aligned}
$$

such that $\langle A\rangle=\phi\langle A\rangle_{\mathcal{P}}$ where $\phi=\left\|\mathcal{P}_{\mathcal{U}}\right\| /\|\mathcal{U}\|$ is the porosity. We use the multiple-scale expansion technique ${ }^{16,17}$ to derive effective continuum scale equations for average concentration $C(\mathbf{x}, t) \equiv\langle c(\mathbf{x}, t)\rangle$. The method postulates that concentration exhibits both large-scale (across the porous material, denoted by the coordinate $\mathbf{x}$ ) and small-scale (inside individual pores, denoted by the coordinate $\mathbf{y}$ ) spatial variability, such that $\mathbf{y}=\epsilon^{-1} \mathbf{x}$ with $\epsilon \ll 1$; the corresponding temporal scales are denoted, respectively, by $t$ and $\tau_{r}=\mathrm{Da} t$ where $\mathrm{Da}$ is the Damköhler number.

Let us introduce a membership (indicator) function $\Pi(\mathbf{x})=\Pi(\mathbf{x} / \epsilon)$,

$$
\Pi(\mathbf{x})= \begin{cases}1, & \mathbf{x} \in \mathcal{P} \\ 0, & \mathbf{x} \in \mathcal{S} .\end{cases}
$$

For the nanoporous materials under consideration, the function $\Pi(\mathbf{x})=\Pi(\mathbf{x} / \epsilon)$ is periodic on the unit cell $\mathcal{U}$. This allows one to define the pore-scale diffusion equation (6b) on the whole unit cell $\mathcal{U}$ (rather than on the multi-connected subdomain $\mathcal{P}_{\mathcal{U}}$ occupied the liquid)

$$
\frac{\partial c}{\partial t}=\nabla \cdot(\Pi \nabla c), \quad \mathbf{y} \in \mathcal{U}, \quad t>0
$$

Replacing the concentration $c(\mathbf{x}, t)$ with $c\left(\mathbf{x}, \mathbf{y}, t, \tau_{r}\right)$ and substituting into Eq. (B3) yields

$$
\begin{aligned}
\frac{\partial c}{\partial t}+\operatorname{Da} \frac{\partial c}{\partial \tau_{r}}= & \nabla_{\mathbf{x}} \cdot\left[\Pi\left(\nabla_{\mathbf{x}} c+\epsilon^{-1} \nabla_{\mathbf{y}} c\right)\right]+\epsilon^{-1} \nabla_{\mathbf{y}} \\
\cdot & {\left[\Pi\left(\nabla_{\mathbf{x}} c+\epsilon^{-1} \nabla_{\mathbf{y}} c\right)\right], \quad \mathbf{y} \in \mathcal{U} . }
\end{aligned}
$$

The interfacial condition (6a) takes the form

$$
-\mathbf{n} \cdot\left(\nabla_{\mathbf{x}} c+\epsilon^{-1} \nabla_{\mathbf{y}} c\right)=\operatorname{Da} q_{\mathrm{m}} \frac{K c}{1+K c}, \quad \mathbf{y} \in \Gamma_{\mathcal{U}} .
$$

In the multiple-scale expansion method, the concentration $c\left(\mathbf{x}, \mathbf{y}, t, \tau_{r}\right)$ is represented by an asymptotic series

$$
c\left(\mathbf{x}, \mathbf{y}, t, \tau_{r}\right)=\sum_{m=0}^{\infty} \epsilon^{m} c_{m}\left(\mathbf{x}, \mathbf{y}, t, \tau_{r}\right) .
$$

Substituting Eq. (B5) into Eq. (B4), approximating the Langmuir adsorption isotherm with a series

$$
\begin{aligned}
\frac{K c}{1+K c}= & 1-\frac{1}{1+K c}=1-\left[1-K\left(c_{0}+\epsilon c_{1}\right)\right. \\
& \left.+K^{2}\left(c_{0}^{2}+2 \epsilon c_{1} c_{0}\right)\right]+\mathcal{O}\left(\epsilon^{2}\right), \\
= & K c_{0}-K^{2} c_{0}^{2}+\epsilon\left(K c_{1}-2 K^{2} c_{0} c_{1}\right)+\mathcal{O}\left(\epsilon^{2}\right),
\end{aligned}
$$

and recalling from Appendix A that $\mathrm{Da} \sim \mathcal{O}\left(\epsilon^{1}\right)$, we obtain 


$$
\begin{aligned}
-\epsilon^{-2} \nabla_{\mathbf{y}} \cdot\left(\Pi \nabla_{\mathbf{y}} c_{0}\right)-\epsilon^{-1}\left\{\nabla_{\mathbf{x}} \cdot\left(\Pi \nabla_{\mathbf{y}} c_{0}\right)\right. \\
\left.\quad+\nabla_{\mathbf{y}} \cdot\left[\Pi\left(\nabla_{\mathbf{y}} c_{1}+\nabla_{\mathbf{x}} c_{0}\right)\right]\right\} \\
+\epsilon^{0}\left\{\frac{\partial c_{0}}{\partial t}-\nabla_{\mathbf{x}} \cdot\left[\Pi\left(\nabla_{\mathbf{x}} c_{0}+\nabla_{\mathbf{y}} c_{1}\right)\right]\right. \\
\left.\quad-\nabla_{\mathbf{y}} \cdot\left[\Pi\left(\nabla_{\mathbf{x}} c_{1}+\nabla_{\mathbf{y}} c_{2}\right)\right]\right\}=\mathcal{O}\left(\epsilon^{1}\right), \quad \mathbf{y} \in \mathcal{U}
\end{aligned}
$$

and

$$
\begin{aligned}
& -\epsilon^{-1} \mathbf{n} \cdot \nabla_{\mathbf{y}} c_{0}-\epsilon^{0}\left[\mathbf{n} \cdot\left(\nabla_{\mathbf{x}} c_{0}+\nabla_{\mathbf{y}} c_{1}\right)\right] \\
& \quad-\epsilon^{1}\left[q_{\mathrm{m}}\left(K c_{0}-K^{2} c_{0}^{2}\right)\right. \\
& \left.\quad+\mathbf{n} \cdot\left(\nabla_{\mathbf{x}} c_{1}+\nabla_{\mathbf{y}} c_{2}\right)\right]=\mathcal{O}\left(\epsilon^{2}\right), \quad \mathbf{y} \in \Gamma_{\mathcal{U}} .
\end{aligned}
$$

Collecting terms of the equal powers of $\epsilon$ yields BVPs for $c_{m}\left(\mathbf{x}, \mathbf{y}, t, \tau_{r}\right)(m=0,1, \ldots)$.

Leading-order term, $\mathcal{O}\left(\epsilon^{-2}\right)$ : Collecting the $\mathcal{O}\left(\epsilon^{-2}\right)$ terms yields a BVP for the leading-order term in expansion (B5)

$$
\nabla_{\mathbf{y}} \cdot\left(\Pi \nabla_{\mathbf{y}} c_{0}\right)=0, \quad \mathbf{y} \in \mathcal{U} ; \quad-\mathbf{n} \cdot \nabla_{\mathbf{y}} c_{0}=0, \quad \mathbf{y} \in \Gamma_{\mathcal{U}} .
$$

This BVP has a trivial solution, which implies that $c_{0}$ is independent of $\mathbf{y}$.

Term of order $\mathcal{O}\left(\epsilon^{-1}\right)$ : Since $\mathrm{Da} \sim \mathcal{O}\left(\epsilon^{1}\right)$, collecting the $\mathcal{O}\left(\epsilon^{-1}\right)$ terms yields a BVP for the first-order term in expansion (B5)

$$
\nabla_{\mathbf{y}} \cdot\left[\Pi\left(\nabla_{\mathbf{x}} c_{0}+\nabla_{\mathbf{y}} c_{1}\right)\right]=0, \quad \mathbf{y} \in \mathcal{U}
$$

subject to the interfacial condition

$$
-\mathbf{n} \cdot\left(\nabla_{\mathbf{x}} c_{0}+\nabla_{\mathbf{y}} c_{1}\right)=0, \quad \mathbf{y} \in \Gamma_{\mathcal{U}}
$$

Equations (B10), which form a BVP for $c_{1}\left(\mathbf{x}, \mathbf{y}, t, \tau_{r}\right)$, define a local problem. It depends only on the geometry of the unit cell. We represent its solution as ${ }^{16,17}$

$$
c_{1}\left(\mathbf{x}, \mathbf{y}, t, \tau_{r}\right)=\chi(\mathbf{y}) \cdot \nabla_{\mathbf{x}} c_{0}\left(\mathbf{x}, t, \tau_{r}\right)+\bar{c}_{1}\left(\mathbf{x}, t, \tau_{r}\right) .
$$

Substituting this into Eq. (B10a) yields an equation for the closure variable (a vector) $\chi(\mathbf{y})$

$$
\nabla_{\mathbf{y}} \cdot\left(\mathbf{I}+\nabla_{\mathbf{y}} \chi\right) \nabla_{\mathbf{x}} c_{0}=0, \quad \mathbf{y} \in \mathcal{P}_{\mathcal{U}}
$$

This equation is subject to $\langle\chi\rangle=0$ and the boundary condition

$$
\mathbf{n} \cdot\left(\mathbf{I}+\nabla_{\mathbf{y}} \chi\right) \nabla_{\mathbf{x}} c_{0}=0, \quad \mathbf{y} \in \Gamma_{\mathcal{U}}
$$

which is obtained by substituting Eq. (B11) into Eq. (B10b).

Terms of order $\mathcal{O}\left(\epsilon^{0}\right)$ : Collecting the terms of order $\epsilon$ (B7) yields

$$
\begin{aligned}
& \frac{\partial c_{0}}{\partial t}-\nabla_{\mathbf{x}} \cdot\left[\Pi\left(\nabla_{\mathbf{x}} c_{0}+\nabla_{\mathbf{y}} c_{1}\right)\right] \\
& \quad-\nabla_{\mathbf{y}} \cdot\left[\Pi\left(\nabla_{\mathbf{x}} c_{1}+\nabla_{\mathbf{y}} c_{2}\right)\right]=0, \quad \mathbf{y} \in \mathcal{U}
\end{aligned}
$$

subject to the interfacial condition

$$
-q_{\mathrm{m}}\left(K c_{0}-K^{2} c_{0}^{2}\right)-\mathbf{n} \cdot\left(\nabla_{\mathbf{x}} c_{1}+\nabla_{\mathbf{y}} c_{2}\right)=0, \quad \mathbf{y} \in \Gamma_{\mathcal{U}}
$$

Integrating over $\mathcal{U}$ with respect to $\mathbf{y}$, applying the interfacial conditions (B14b), and accounting for the periodicity of $\Pi(\mathbf{y})$ on the boundary of the unit cell $\mathcal{U}$, we obtain

$$
\begin{gathered}
\frac{\partial c_{0}}{\partial t}-\nabla_{\mathbf{x}} \cdot\left(\phi^{-1}\left\langle\left(\mathbf{I}+\nabla_{\mathbf{y}} \chi\right)\right\rangle \nabla_{\mathbf{x}} c_{0}\right) \\
+\gamma_{\mathrm{eff}} q_{\mathrm{m}}\left(K c_{0}-K^{2} c_{0}^{2}\right)=0
\end{gathered}
$$

where $\gamma_{\text {eff }}$ is defined in Eq. (9), $\phi$ is the porosity and effective diffusion coefficient $\mathbf{D}_{\text {eff }} / D=\left\langle\left(\mathbf{I}+\nabla_{\mathbf{y}} \chi\right)\right\rangle$ is defined in (10).

Rewriting the linearized form of the Langmuir isotherm, $K c_{0}-K^{2} c_{0}^{2}$, in its original form, $K c_{0} /\left(1+K c_{0}\right)$; approximating the solute concentration $c \approx c_{0}$; and defining its average over the cell as $C(\mathbf{x}, t) \equiv\langle c(\mathbf{x}, t)\rangle$ leads to the homogenized continuum-scale diffusion-reaction equation for the average concentration (8).

${ }^{1}$ J. S. Seo, D. Whang, H. Lee, S. Im Jun, J. Oh, Y. J. Jeon, and K. Kim, “A homochiral metal-organic porous material for enantioselective separation and catalysis," Nature 404, 982-986 (2000).

${ }^{2}$ C.-M. Yang, Y.-J. Kim, M. Endo, H. Kanoh, M. Yudasaka, S. Iijima, and K. Kaneko, "Nanowindow-regulated specific capacitance of supercapacitor electrodes of single-wall carbon nanohorns," J. Am. Chem Soc. 129, 20-21 (2007).

${ }^{3}$ T. Ohkubo, J. Miyawaki, K. Kaneko, R. Ryoo, and N. A. Seaton, "Adsorption properties of templated mesoporous carbon (CMK-1) for nitrogen and supercritical methane experiment and GCMC simulation," J. Phys. Chem. B 106, 6523-6528 (2002).

${ }^{4}$ T. A. Desai, D. J. Hansford, L. Kulinsky, A. H. Nashat, G. Rasi, J. Tu, Y. Wang, M. Zhang, and M. Ferrari, "Nanopore technology for biomedical applications," Biomed. Microdevices 2, 11-40 (1999).

${ }^{5}$ S. P. Adiga, L. A. Curtiss, J. W. Elam, M. J. Pellin, C.-C. Shih, C.-M. Shih, S.-J. Lin, Y.-Y. Su, S. D. Gittard, and J. Zhang, "Nanoporous materials for biomedical devices," JOM 60, 26-32 (2008).

${ }^{6}$ A. J. Rosenbloom, D. M. Sipe, Y. Shishkin, Y. Ke, R. P. Devaty, and W. J. Choyke, "Nanoporous SiC: A candidate semi-permeable material for biomedical applications," Biomed. Microdevices 6(4), 261-267 (2004).

7"Basic research needs for electric energy storage," Technical Report (United State Department of Energy, 2007).

${ }^{8}$ S. Kondrat, C. R. Pérez, V. Presser, Y. Gogotsi, and A. A. Kornyshev, "Effect of pore size and its dispersity on the energy storage in nanoporous supercapacitors," Energy Environ. Sci. 5, 6474-6479 (2012).

${ }^{9}$ M. P. Klein, B. W. Jacobs, M. D. Ong, S. J. Fares, D. B. Robinson, V. Stavila, G. J. Wagner, and I. Arslan, "Three-dimensional pore evolution of nanoporous metal particles for energy storage," J. Am. Chem. Soc. 133, 9144-9147 (2011).

${ }^{10}$ M. E. Davis, "Ordered porous materials for emerging applications," Nature 417, 813-821 (2002).

${ }^{11}$ I. Moriguchi, M. Honda, T. Ohkubo, Y. Mawatari, and Y. Teraoka, "Adsorption and photocatalytic decomposition of methylene blue on mesoporous metallosilicates," Catal. Today 90, 297-303 (2004).

${ }^{12}$ H. Yamada, H. Nakamura, F. Nakahara, I. Moriguchi, and T. Kudo, "Electrochemical study of high electrochemical double layer capacitance of ordered porous carbons with both meso/macropores and micropores," J. Phys. Chem. C 111, 227-233 (2007).

${ }^{13}$ L. L. Zhang and X. S. Zhao, "Carbon-based materials as supercapacitor electrodes," Chem. Soc. Rev. 38, 2520-2531 (2009).

${ }^{14}$ G. Severino, G. Dagan, and C. J. van Duijn, "A note on transport of a pulse of nonlinearly reactive solute in a heterogeneous formation," J. Comput. Geosci. 4, 275-286 (2000).

${ }^{15} \mathrm{~S}$. Whitaker, The Method of Volume Averaging (Kluwer Academic Publishers, Netherlands, 1999). 
${ }^{16} \mathrm{U}$. Hornung, Homogenization and Porous Media (Springer, New York, 1997).

${ }^{17}$ I. Battiato and D. M. Tartakovsky, "Applicability regimes for macroscopic models of reactive transport in porous media," J. Contam. Hydrol. 120-121, 18-26 (2011).

${ }^{18}$ G. Allaire, A. Mikelić, and A. Piatnitski, "Homogenization approach to the dispersion theory for reactive transport through porous media," SIAM J. Math. Anal. 42, 125-144 (2010).

${ }^{19}$ I. Moriguchi, Y. Shono, H. Yamada, and T. Kudo, "Colloidal crystalderived nanoporous electrode materials of cut SWNTs-assembly and $\mathrm{TiO}_{2}$ /SWNTs nanocomposite," J. Phys. Chem. B 112, 14560-14565 (2008).

${ }^{20}$ C. G. Enfield, C. C. Harlin, and B. E. Bledsoe, "Comparison of five kinetic models for orthophosphate reactions in mineral soils," Soil Sci. Soc. Am. J. 40, 243-249 (1976).

${ }^{21}$ C. J. Van Duijn and P. Knabner, "Travelling waves in the transport of reactive solutes through porous media: Adsorption and binary ion exchangePart 1," Transp. Porous Media 8, 167-194 (1992).

${ }^{22}$ W. J. Weber, P. M. McGinley, and L. E. Katz, "Sorption phenomena in subsurface systems: Concepts, models and effects on contaminant fate and transport," Water Res. 25, 499-528 (1991).

${ }^{23}$ G. Allaire, R. Brizzi, A. Mikelić, and A. Piatnitski, "Two-scale expansion with drift approach to the Taylor dispersion for reactive transport through porous media," Chem. Engrg. Sci. 65, 2292-2300 (2010).

${ }^{24}$ S. Azizian, "Kinetic models of sorption: A theoretical analysis," J. Colloid Interface Sci. 276, 47-52 (2004).

${ }^{25}$ C. Cheung, J. Porter, and G. McKay, "Sorption kinetics for the removal of copper and zinc from effluents using bone char," Sep. Purif. Technol. 19, 55-64 (2000).
${ }^{26}$ Z. Reddad, C. Gerente, Y. Andres, and P. Le Cloirec, "Adsorption of several metal ions onto a low-cost biosorbent: Kinetic and equilibrium studies," Environ. Sci. Technol. 36, 2067-2073 (2002).

${ }^{27} \mathrm{~S}$. Saraji, L. Goual, and M. Piri, "Adsorption of asphaltenes in porous media under flow conditions," Energy Fuels 24, 6009-6017 (2010).

${ }^{28}$ C. A. Grattoni, R. A. Dawe, and M. S. Bidner, "On the simultaneous determination of dispersion and nonlinear adsorption parameters from displacement tests by using numerical models and optimisation techniques," Adv. Water Resour. 16, 127-135 (1993).

${ }^{29}$ J. Bear and A.-D. Cheng, Modeling Groundwater Flow and Contaminant Transport (Springer, 2010), Vol. 23.

${ }^{30}$ W. Plazinski, W. Rudzinski, and A. Plazinska, "Theoretical models of sorption kinetics including a surface reaction mechanism: A review," Adv. Colloid Interface Sci. 152, 2-13 (2009).

${ }^{31}$ Y.-S. Ho and G. McKay, "Pseudo-second order model for sorption processes," Process Biochem. 34, 451-465 (1999).

${ }^{32}$ G. Limousin, J.-P. Gaudet, L. Charlet, S. Szenknect, V. Barthes, and M. Krimissa, "Sorption isotherms: A review on physical bases, modeling and measurement," Appl. Geochem. 22, 249-275 (2007).

${ }^{33}$ M. J. Pellin, P. C. Stair, G. Xiong, J. W. Elam, J. Birrell, L. Curtiss, S. M. George, C. Y. Han, L. Iton, and H. Kung, "Mesoporous catalytic membranes: Synthetic control of pore size and wall composition," Catal. Lett. 102, 127-130 (2005).

${ }^{34}$ D. E. Giammar and J. G. Hering, "Time scales for sorption-desorption and surface precipitation of uranyl on goethite," Environ. Sci. Technol. 35, 3332-3337 (2001)

${ }^{35}$ K. L. Kostka, M. D. Radcliffe, and E. von Meerwall, "Diffusion coefficients of methylene blue and thioflavin T dyes in methanol solution," J. Phys. Chem. 96, 2289-2292 (1992) 